Ann. A beille, I968, 11 (2), II7-I24.

\title{
ACTION D'UN EXTRAIT TOTAL DE RACINE DE GALANGA SUR BACILLUS LARVAE. ESSAI DE TRAITEMENT DE LA LOQUE AMERICAINE
}

\author{
A. BRIZARD, P. SAURAT, J. CHANTAL, P. LAVIE \\ Avec la collaboration technique de Germaine Delorme, J. Albisetti, M. Gonnet \\ Laboratoire des Services de Parasitologie et des Maladies légalement contagieuses, \\ École nationale vétérinaire, 31 - Toulouse \\ Station expérimentale d'Apiculture, \\ Centre de Recherches agronomiques du Sud-Est, 84 - Montfavet \\ Institut national de la Recherche agronomique
}

\section{SOMMAIRE}

Les expériences in zitro montrent que des extraits totaux de racine de Galanga (Alpinia officinarum) ont vis-à-vis de Bacillus larvae une activité bactériostatique à des concentrations supérieures à $\mathrm{I} / 25000$ et seulement " bactériofreinatrice " à I/50 000 et I/100 000. Ces extraits sont incapables de débarrasser les colonies d'abeilles de la Loque américaine à une posologie comparable et même supérieure à celle retenue pour les antibiotiques usuels.

\section{INTRODUC'TION}

En I960, l'un de nous décrivit les propriétés antibactériennes de la propolis (LAVIE, I960). En I963, constatant que l'activité bactériostatique de cette " résine " est proportionnelle aux taux de flavonoïdes qu'elle contient, il en isola une substance, la $3,5,7$ - trihydroxyflavone ou galangine, dont il étudia le pouvoir inhibiteur sur Bacillus subtilis caron, Bacillus alvei, Proteus vulgaris, Salmonella gallinarum $\mathbf{n}^{0} 38$, Salmonella Dublin 4-54, Escherichia coli O 55 B 5 Ec 5 4OI, Escherichia coli DEMEREK et FANO (VILILANUfiva et al., I964).

Les résultats obtenus l'ayant amené à penser que cette flavone pourrait jouer un rôle dans la résistance plus ou moins grande des colonies d'abeilles aux maladies bactériennes, nous avons voulu vérifier cette hypothèse de travail. Les quantités de galangine dont nous pouvions disposer nous ont imposé de limiter notre expé- 
rimentation de deux côtés. Tout d'abord, faute de pouvoir se procurer suffisamment de galangine de synthèse ou de galangine isolée à partir de la propolis, nous avons testé un extrait total de racine de Galanga (Alpina officinarum) (Schraurstatrer, I948, I95 I). Cet extrait renferme de la galangine, mais également d'autres substances bactériostatiques. Il n'a pas été possible d'évaluer la concentration en galangine dans le mélange, car il est très difficile de connaître dans quelles proportions les flavonols sont libérés de leurs glycosides au cours de l'extraction (SwAIN, 1962). 1)'autre part, la quantité d'extrait dont nous disposions nous a obligés à limiter nos essais, et nous avons choisi, pour réaliser ceux-ci, la Loque américaine, en raison de sa gravité, de son étiologie bien établie et de la constance de son évolution.

\section{MÉTHODES ET RÉSULTATS}

Deux séries de recherches furent entreprises : 1'une avait pour but d'établir in vitro le pouvoir antibiotique de l'extrait sur l'agent causal, Bacillus larvae; 1'autre visait à contrôler 1'action de la substance sur l'évolution de la maladie.

\section{I. - REChERChES "IN VITRO ".}

\section{POUVOIR ANTIBACTÉRIEN SUR "BACILI,US LARVAE »}

Il a été étudié sur 2 souches, dont l'une provenait d'un rucher où furent conduits les essais thérapeutiques.

Assez peu aisé à cultiver et à conserver, $B$. lavvac fut isolé et identifié après ensemencement et repiquage sur bouillon ou sur gélose de Holst et Sturtevant.

Pratiquement insoluble dans l'eau, l'extrait fut mis en solution, d'une part dans la diméthylformamide, d'autre part dans du DMSO (1) à des concentrations de I/IOO, I/I 000, I/IO 000, I/I00 000.

Dans une première expérience, le pouvoir antibactérien de la substance fut mis en évidence par la méthode bien connue des disques par diffusion sur gélose. Nous n'entreprendrons donc pas ici d'en décrire les détails et nous nous bornerons à indiquer qu'une nette inhibition fut obtenue avec la solution à I/ I OOO, alors que les dilutions supérieures ne paraissaient gêner en rien le développement microbien. Ce résultat ayant été jugé insuffisant, une deuxième expérience fut alors entreprise pour mesurer de façon plus exacte l'activité antibactérienne de l'extrait vis-à-vis de Bacillus larvac. Au couts d'un premier essai, le bacille fut cultivé sur bouillon additionné de jaune d'œuf dans la proportion de I pour Io. I a substance en solution au I/Ioo dans le diméthylformamide fut ajoutée dans 5 tubes à ce bouillon enrichi de façon à obtenir des concentrations successives de I/I 000, I/IO 000, I/IOO 000 , I/I $000000, \mathrm{I} / \mathrm{I} 0000000$. Tous ces tubes ainsi que 2 témoins furent alors ensemencés à l'aide de 3 gouttes d'une culture de $48 \mathrm{~h}$ de $B$. larvac sur bouillon à l'ouf.

Les résultats furent enregistrés après $48 \mathrm{~h}$ et $72 \mathrm{~h}$ d'incubation à $37^{\circ}$, par examen microscopique, 1'appréciation directe ne pouvant être effectuée en raison du trouble normal du milieu dû à l'œuf. On peut établir ainsi que l'extrait de racine

(1) D.M.S.O. : diméthylsulfoxyde. 
de Galanga possède un pouvoir inhibiteur à une dilution comprise entre I/Io ooo et I/roo 000, mais qu'aux dilutions supérieures la quantité de germes trouvée est la même dans les différents tubes.

Un deuxième titrage fut alors réalisé pour obtenir encore une plus grande précision. Afin de pouvoir suivre plus facilement les résultats, les dilutions et les ensemencements furent effectués sur gélose de Holst et Sturtevant maintenue en surfusion puis inclinée, et 5 tubes furent préparés renfermant l'extrait à des concentrations de I/6 250, I/I2 500, I/25 000, I/50 000, I/I00 000. I,es ensemencements furent réalisés comme dans le cas précédent et les résultats appréciés par examen des colonies développées. On peut ainsi noter qu'aucune colonie ne se forma dans les tubes de concentration $\mathrm{I} / 625^{\circ}, \mathrm{I} / \mathrm{I} 25^{00} \mathrm{I} / 25$ ooo. Par contre, aux dilutions de $\mathrm{I} / 50000$ et I/I00 000, un certain nombre de colonies se développèrent mais plus tardivement et plus faiblement que dans les tubes témoins.

De cette expérimentation il résulte donc:

I $^{\circ}$ que l'extrait total de racine de Galanga a une activité antibactérienne sur Bacillus larvae jusqu'à une dilution de I/I00 ooo ;

$2^{\circ}$ que cette activité est en rapport avec un effet bactériostatique à des concentrations supérieures à $\mathrm{I} / 25000$;

$3^{\circ}$ qu'elle est seulement "bactériofreinatrice " de I/50 000 à I/I00 000 .

Notons que cette activité antibactérienne est plus faible que celle des antibiotiques classiques dont l'action se manifeste à des dilutions de l'ordre du millionième. Cependant, il est bon de rappeler que nous avons testé un extrait total de racine de Galanga non purifié et que celui-ci est beaucoup moins actif que 1a 3, 5, 7-trihydroxyflavone elle-même.

\section{II. - RECHERCHES «IN VIVO ».}

ACTIVITÉ 'THÉRAPEUTIQUF DE L'EXTTRATT DE RACINE DE GALANGA VIS-A-VIS DE I.A LOQUE AMÉRICAINE

Pouvait-on penser, en fonction de ces résultats de laboratoire, que la substance était capable, à dose convenable, d'enrayer le développement de la Loque américaine? La deuxième série de recherches permet de répondre à cette question.

Faute d'une quantité suffisante de produit, les essais durent être limités en nombre et la posologie maintenue dans les expériences préliminaires à des doses que nous aurions voulues plus fortes.

Pour son administration, l'extrait fut dissous à une concentration de 6 p. I ooo dans une solution décinormale de carbonate de soude et celle-ci pulvérisée directement sur le couvain. Les colonies traitées se trouvaient dans des ruches " Dadant».

Les essais ont été étalés sur deux années : i965 et i966. Comme dans toutes nos expérimentations de même nature, le diagnostic fut toujours vérifié au laboratoire par la mise en évidence de Bacillus larvae et l'évolution de la maladie suivie aussi attentivement que possible avant, pendant et après le traitement.

\section{A. - Expériences conduites en 1965}

Elles ont consisté, avec la petite quantité d'extrait dont nous disposions, à traiter 3 colonies préalablement infectées par l'introduction dans chaque ruche d'un 
ou deux cadres de couvain très fortement loqueux provenant de colonies atteintes naturellement. Un témoin reçut, dans le même temps et selon une technique identique, des quantités de solvant (solution décinormale de carbonate de soude) égales à celles ayant servi à dissoudre les plus fortes doses de substance.

I. Colonie $64 / 6$.

Assez faible en début de saison (mai) cette colonie présente des traces d'une atteinte loqueuse de l'année précédente (écailles). Renforcée par réunion avec une autre colonie, remérage et apport de couvain sain, le développement du processus pathologique est accéléré par l'introduction, fin mai et début juin, de I rayon de couvain sur lequel la loque est largement répandue. A la suite de quoi la maladie prend une extension rapide.

Traitement.

$$
\text { dose et rythme : le } \begin{aligned}
\mathrm{I} 4-6 & =320 \mathrm{mg} \text { d'extrait } \\
23-6 & =240 \mathrm{mg} \\
2-7 & =600 \mathrm{mg}
\end{aligned}
$$

soit au total I,I6o g d'extrait distribué en 3 fois, à une semaine d'intervalle.

Observations.

en $\mathbf{I}_{965}\left\{\begin{aligned} \text { le } 23-7 & \left\{\begin{array}{l}\text { ponte régulière } \\ \text { amélioration de l'état sanitaire }\end{array}\right. \\ \text { le } 3-8: \text { persistance de l'amélioration mais, aussi, de la maladie } & \\ \text { le ro-9 } & \left\{\begin{array}{l}\text { couvain sur } 8 \text { cadres } \\ 6 \text { à ro larves filantes par rayon } \\ B . \text { lavvae }+\end{array}\right.\end{aligned}\right.$

le I8-I I : récolte totale : I2,500 $\mathrm{kg}$

en Ig66 le I8-4 $\left\{\begin{array}{l}\text { couvain sur } 4 \text { cadres } \\ \text { mise en évidence d'une larve operculée } \\ \text { filante : Bacillus larvae }{ }^{+}\end{array}\right.$

\section{Conclusion.}

Malgré une amélioration très nette, due sans doute à ce que la colonie a été renforcée et a reçu une jeune reine, la guérison complète n'a pas été obtenue. On notera cependant que la mise en ouvre tardive du traitement (fin juin, début juillet) peut expliquer en partie - le fait est bien connu (BRIZARD et al.) - son échec.

\section{Colonie 64/9.}

Cette colonie très forte possède 7 grands cadres de couvain, une population adulte très nombreuse et active, et d'abondantes réserves. Elle est contaminée le 20 mai par introduction de 2 cadres de couvain très largement atteint par la maladie. A la suite de quoi la Loque se propage sur les rayons indemnes. Cette colonie reçoit deux traitements.

\section{Ier traitement.}

Il consiste en une seule pulvérisation le ${ }^{4}-6$ sur le couvain de $320 \mathrm{mg}$ de substance.

Observations. Le $23-7$, la maladie frappe les larves dans la proportion de Io à I5 p. roo suivant les rayons. 


\section{$2^{\mathrm{e}}$ traitement.}

Il est effectué 50 jours après le premier, suivant la même technique mais 3 doses d'extrait sont administrées : le $3-8=5$ I0 $\mathrm{mg}$; I0- $8=5$ I0 $\mathrm{mg} ;$ I $7-8=300 \mathrm{mg}$, soit au total, I,320 g distribué en 3 fois, à une semaine d'intervalle.

\section{Observations.}

Le 3-8, lors de la première pulvérisation, la Loque est installée sur 9 rayons.

Le I7-8, sur certains rayons, le nombre des larves malades paraît en diminution mais le nettoyage des cellules se fait mal et plusieurs d'entre elles sont comblées par les ouvrières à l'aide d'un bouchon de cire.

Le Io-9, la maladie n'a pas disparu.

Sur les cadres d'inoculation elle atteint 50 p. Ioo des larves. Aucune récolte.

\section{Conclusion.}

Malgré 4 doses d'extrait (au total r,630 g), dont 3 administrées dans les conditions classiques d'un traitement par antibiotique, et malgré la force de la colonie, la maladie n'a pas disparu.

On notera cependant que, comme dans le cas de la précédente colonie, le traitement a été appliqué tardivement (début août).

\section{Colonie $64 / 10$.}

Il s'agit, là aussi, d'une colonie forte qui possède un couvain abondant sur 5 rayons et de bonnes réserves. Ëlle est contaminée le 20 mai, lors de la visite qui permet ces constatations, par introduction de 2 rayons de couvain où les larves malades sont très nombreuses.

Traitement. Dose. Le produit faisant défaut une seule dose de $320 \mathrm{mg}$ est donnée le I4-6.

Observations. cinq semaines plus tard la visite de la colonie toujours forte et riche en couvain révèle une extension de la L,oque qui frappe, suivant les rayons, Io à 15 p. Ioo des larves.

Conclusion. La dose unique de $320 \mathrm{mg}$ n'a pas empêché le développement de la maladie.

\section{Traitement de comparaison.}

Pour pouvoir mieux apprécier l'action de l'extrait de racine de Galanga par rapport à celle de médicaments reconnus efficaces, nous traitons cette même colonie les 3, Io et I7 août, donc très tard dans la saison, par aspersion des rayons avec $0,50 \mathrm{~g}$ chaque fois de chlorhydrate de tétracycline dissous dans $\mathrm{I} / 3$ de litre de sirop de sucre. Une visite pratiquée en septembre montre qu'il n'y a plus de larves fraîchement atteintes et que, seules, persistent 4 à 6 écailles loqueuses par rayon. Au printemps ig66 la colonie paraît complètement assainie et des examens successifs ne permettent pas de dépister la moindre trace de L,oque.

Il est important de souligner que dans le même temps, le traitement de la colonie 64/9 par extrait de Galanga (les 3, Io et I7 août) a été incapable de faire disparaître la maladie et ce malgré une première dose de $320 \mathrm{mg}$ distribuée en juin. 


\section{Colonie B/14.}

Dans cette série d'expériences préliminaires, une colonie (B/I4) est gardée comme témoin à côté des autres. Elle reçoit, pour que 1'on puisse éliminer une éventuelle action du solvant, 3 fois à 8 jours d'intervalle et aux mêmes dates que 1a 64/9: d'abord, en juin, $54 \mathrm{ml}$ puis, en août, 3 fois à 8 jours d'intervalle, $85 \mathrm{ml}$ de carbonate de soude en solution décinormale:

- en juillet la L,oque s'est étendue à tous les rayons,

- en septembre on trouve 7 à 8 p. Ioo de larves operculées présentant les caractères typiques de la Loque américaine (confirmation par mise en évidence de $B$. larvae),

- en octobre cependant, les ouvrières ont bien nettoyé les rayons et la population et la récolte sont suffisamment importantes pour permettre l'hivernage. Mais, en avril rg66, la maladie repart de plus belle. Le solvant n'a donc pas permis à cette colonie de se débarrasser de la maladie.

\section{B. - Expériences conduites en 1966}

Conçues dans le même esprit et pour atteindre le même but que celles de 65 , elles ont été réalisées :

I $^{\circ}$ avec des doses plus régulièrement élevées,

$2^{\circ}$ de façon plus précoce afin d'éliminer comme cause d'échec l'insuffisance des traitements tardifs,

$3^{0}$ sur 3 colonies, dont une seule contaminée artificiellement, l'évolution chez les deux autres infectées expérimentalement l'année précédente s'étant faite naturellement en Ig66 du fait de leur non-guérison. Une colonie témoin fut gardée à laquelle le solvant fut administré seul.

Une suite de circonstances défavorables ne nous ayant pas permis d'effectuer les contrôles habituels pendant et après le traitement, c'est dès le début de la saison apicole suivante que les résultats furent enregistrés.

\section{Colonie $64 / 10$.}

Traitée par l'extrait de racine da Galanga en I965, cette colonie doit, pour pouvoir survivre pendant l'hiver, resevoir une médication à base de chlorhydrate de tétracycline. Bien assainie à 1'automne I965 elle ne présente absolument aucune trace de maladie le i 8 avril et le 5 mai I956. I) force moyenne elle est infectée par introluction de 2 cadres de couvain loqueux. A la suite de quoi la maladie se développe sur les rayons voisins.

Traitement. I g de la substance est administré 3 fois (autotal $3 \mathrm{~g}$ ) à une semaine d'intervalle les 2 I et 27 mai et le 3 juin.

Observations.

20-3-57. Population plutôt faible, couvain sur + rayons. Nombreuses écailles de Loque américaine, traces incontestables du développement de la maladie en Ig66.

13-4-67. Bon développement de la colonie. Couvain sur 5 cadres mais 35 à $40 \mathrm{p}$. Ioo des larves présentent les signes d'une Loque américaine récente (B. larvae $\left.{ }^{+}\right)$. 
Conchtsion. Échec du traitement.

2. Colonie $64 / 9$.

Déjà traitée sans succès en 1965 avec l'extrait de racine de Galanga (I,32 g) la 64-9, malgré uñ thérapeutique au sulfathiazol à vrai dire trop tardive, présente le 5 mai I955 de nonoreuses traces de la maladie (écailles). Le I3 une amélioration spontanée de l'état sanitaire est constatée à la suite du nettoyage par les ouvrières, mais la colonie demeure faible.

Traitement. Il est conduit dans les mêmes conditions exactement et aux mêmes dates que celui de la 64-Io: soit $3 \mathrm{~g}$ au total d'extrait.

Observations.

20-3-57. L'hivernage a été mauvais : beaucoup de moisissures sur les rayons. Population faible mais bonne reine, 2 cadres avec peu de couvain. Très grande quantité d'écailles loqueuses sur 7 rayons et sur 2 des larves filantes $\left(B\right.$. larvae $\left.{ }^{+}\right)$.

I2-4-67. La colonie est trouvée morte.

Conclusion. Échec du traitement.

3. Colonie B/14.

Elle a servi d z témoin en I965. Au printemps Ig66 la Loque reprend son évcilutirn.

I8-4-57. Colonie de force moyenne. Couvain sur 3 cadres. Nombreuses écailles sur un cadre non occupś et larves filantes sur les autres.

5-5-67. Colonie moyenne. Couvain sur 4 cadres. 7 p. Ioo environ de larves malades sur tous les rayons.

Traitement. Pour étudier les effets d'une dose massive et vérifier en même temps si les quantités précédentes administrées en I seule fois ne donneraient pas un meilleur résultat, $3 \mathrm{~g}$ de produit sont pulvérisés sur les rayons dans la quantité de solvant appropriée $(75 \mathrm{ml})$ le $2 \mathrm{I}-5-66$.

Obseriations.

20-3-67. Colonie très faible. Couvain sur 2 cadres. Écailles loqueuses.

I3-4-57. Population très faible. 2 petits cađres de couvain. Écailles loqueuses et larves filantes (B. larvae ${ }^{+}$).

Conclusion. Échec du traitement.

4. Colonic 64/6.

Gardée comme témoin, elle reçoit les 21 et $27-5$ et le 3-6-66, $25 \mathrm{ml}$ chaque fois de solvant. Assez forte, elle ne présente au mois de mai qu'une atteinte légère de Loque.

Observations. 20-3-67. Population normale. Couvain sur 2 cadres. Écailles loqueuses nombreuses : larves atteintes récemment.

Conclusion. Solvant sans effet apparent sur 1a maladie. 


\section{SYNTHÈSE DES RÉSULTATS ET CONCLUSION GÉNÉRALE}

\section{Expériences in vitro}

Elles ont montré que l'extrait total de racine de Galanga a sur B. lavvae une activité antibactérienne de nature bactériostatique à des concentrations supérieures à $\mathrm{I} / 25000$ et seulement bactériofreinatrice à $\mathrm{I} / 50$ o0o et $\mathrm{I} / \mathrm{I} 00000$.

\section{Expériences in vivo}

Lors des 6 essais réalisés avec cette substance une seule amélioration (en Ig65 sur la colonie 64-6), mais non une guérison, fut constatée. Tous les autres traitements se sont soldés par des échecs, même lorsque ils ont été pratiqués à une époque favorable et avec une posologie que l'on peut considérer comme forte par rapport aux antibiotiques classiques. La dose de $3 \mathrm{~g}$, administrée en une seule fois, ne semble pas avoir donné de meilleurs résultats que celle de $\mathrm{I} g$ répétée 3 fois.

Quant au solvant (solution décinormale de $\mathrm{CO}_{3} \mathrm{Na}_{2}$ ) il ne paraît avoir aucune influence sur l'évolution de la L Loque.

En conclusion nous pouvons donc dire que l'extrait total de racine de Galanga exerce sur $B$. larvae une activité antibactérienne prouvée par le laboratoire, mais qu'à la dose de $3 \mathrm{~g}$ par colonie, distribuée en 3 fois ou en une seule, la substance est manifestement incapable d'enrayer le développement de la Loque américaine.

\section{Rę̧u pour publication en mars 1969.}

\section{SUMMARY}

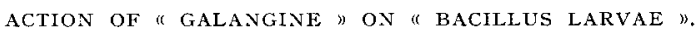

The experiments in vitro show that total extracts of Galanga roots (Alpinia officinarum) have a bacteristatic action on Bacillus lavvae at concentrations superior to $\mathrm{I} / 25.000$ and only a slowing down action "bactériofreinatrice " at $\mathbf{r} / 50.000$ and $\mathrm{r} / \mathrm{ro0}$. ooo. The extracts are unable to rid the bee settlements of the american foul-brood with a posology comparable and even superior to that adopted for the usual antibiotics. The in vivo treatments did not succeed, even when they have were effected at a favourable time and with a posology which can be considered as strong, when compared to classical antibiotics. A dose of $3 \mathrm{~g}$ given at once does not seem to have given better results than a dose of $I \mathrm{~g}$ repeated three times.

\section{RÉFÉRENCES BIBLIOGRAPHIQUES}

Brizard A., Albisetti J., ig66. Les problèmes de pathologie des abeilles dans la forêt landaise. Ann. Abeille, 9 (4), 359-374.

LAvie P., I960. Les substances antibactériennes dans la colonie d'abeilles (Apis mellifica L.) Thèse Sci. nat. Paris.

Schaufstatter F., Experietian 4, 485.

Schraufstatter E., 195I. U. S. Patent $\mathrm{n}^{\circ} 255^{\circ} 269$. Chem. abstr., 45, $7723 \mathrm{f}$.

Swain T., I962. The chemistry of favonoid compounds, 531. Pergamon Press, Londres.

Villanueva V. R., Bogdanovsky D., Barbier M., Gonnet M., Lavie P., I964. Sur l'isolement et l'identification de la 35 , 7-trihydroxyflavone (galangine) à partir de la propolis. Ann. Inst. Pasteur, 106, $292-302$. 